\title{
A new class of monomial bent functions
}

\author{
Anne Canteaut $^{\mathrm{a}}$, Pascale Charpin ${ }^{\mathrm{a}, *}$, Gohar M. Kyureghyan ${ }^{\mathrm{b}}$ \\ a INRIA projet CODES, B.P. 105, 78153 Le Chesnay Cedex, France \\ ${ }^{\mathrm{b}}$ Department of Mathematics, Otto-von-Guericke University of Magdeburg, Universitätsplatz 2, \\ 39106 Magdeburg, Germany
}

Received 19 June 2006; revised 1 February 2007

Available online 12 March 2007

Communicated by Harald Niederreiter

\begin{abstract}
We study the Boolean functions $f_{\lambda}: \mathbf{F}_{2^{n}} \rightarrow \mathbf{F}_{2}, n=6 r$, of the form $f(x)=\operatorname{Tr}\left(\lambda x^{d}\right)$ with $d=2^{2 r}+$ $2^{r}+1$ and $\lambda \in \mathbf{F}_{2^{n}}$. Our main result is the characterization of those $\lambda$ for which $f_{\lambda}$ are bent. We show also that the set of these cubic bent functions contains a subset, which with the constantly zero function forms a vector space of dimension $2 r$ over $\mathbf{F}_{2}$. Further we determine the Walsh spectra of some related quadratic functions, the derivatives of the functions $f_{\lambda}$.
\end{abstract}

(c) 2007 Elsevier Inc. All rights reserved.

Keywords: Boolean function; Bent function; Monomial function; Cubic function; Quadratic function; Derivatives of Boolean functions; Permutation polynomials

\section{Introduction}

A number of recent papers are devoted to the description of new classes of bent functions. One of the main purpose is to determine bent functions which do not belong to a previously known class. For instance, in [4] are constructed non-normal bent functions and in [8] bent functions are obtained by concatenating quadratic functions. Another goal is to find new expressions of bent functions over finite fields. It is essentially the expressions by means of trace-functions which are considered in $[13,17,18]$. More generally, any polynomial $P(x)$ in $\mathbf{F}_{2^{n}}[x]$ can be viewed as a function with $n$ inputs and $n$ outputs. The properties of $P$ are then studied by means of

\footnotetext{
* Corresponding author.

E-mail addresses: anne.canteaut@inria.fr (A. Canteaut), pascale.charpin@inria.fr (P. Charpin), gohar.kyureghyan@mathematik.uni-magdeburg.de (G.M. Kyureghyan).
} 
their $2^{n}$ component functions, the Boolean functions $x \mapsto \operatorname{Tr}(\lambda P(x))$ where $\operatorname{Tr}$ is the trace function from $\mathbf{F}_{2^{n}}$ to $\mathbf{F}_{2}$. Determining the weights of these functions is of great interest in coding theory and cryptography (see [1,13] and [7], for instance).

Our paper is a contribution to the study of the so-called monomial bent functions. The monomial Boolean functions $\mathbf{F}_{2^{n}}$ are those that can be expressed as $x \mapsto \operatorname{Tr}\left(\lambda x^{d}\right)$ for some $\lambda \in \mathbf{F}_{2^{n}}$ and an integer $d$. The characterization of the exponents $d$ and the corresponding $\lambda$ defining a bent monomial function on $\mathbf{F}_{2^{n}}$ is a difficult open problem. In this paper we study the monomial Boolean functions on fields $\mathbf{F}_{2^{n}}$ with $n=6 r(r>1)$ given by

$$
f_{\lambda}(x)=\operatorname{Tr}\left(\lambda x^{d}\right), \quad d=2^{2 r}+2^{r}+1, \lambda \in \mathbf{F}_{2^{n}}^{*}
$$

We first prove that the weight of $f_{\lambda}$ takes only three values when $\lambda$ runs through $\mathbf{F}_{2^{n}}^{*}$, one of them corresponding to the bent case (Theorem 1). We later describe the set of those $\lambda$ such that $f_{\lambda}$ is bent and we prove that these functions are Maiorana-McFarland bent functions. Moreover, we show that a part of these functions form a subspace of dimension $2 r$ of Boolean bent functions on $\mathbf{F}_{2^{n}}$. All these $2^{2 r}-1$ bent functions are cubic (Theorem 3 ).

The functions $f_{\lambda}$ which are not bent appear as a concatenation of quadratic functions on $\mathbf{F}_{2^{3 r}}$ (Theorem 2). We derive a divisibility property of their Walsh spectra (Corollary 1). In Section 3.4 we state some open problems on the Walsh spectra of these functions.

Many properties of a Boolean function are connected with the properties of its derivatives. For example, the derivatives of a Boolean function are used to obtain lower bounds on the nonlinearity profile [5]. The derivatives are also involved in the computations of several criteria about the optimality of a Boolean function for the cryptographic applications (see [6] and, for instance, [3]). In Section 4, we study the derivatives of a bent and non-bent function $f_{\lambda}$. We show that the Walsh transform of such a derivative takes either the values $\left\{0, \pm 2^{5 r}\right\}$ or the values $\left\{0, \pm 2^{4 r}\right\}$ (Theorem 4).

\section{Preliminaries}

In the whole paper, $\alpha$ is a primitive element of $\mathbf{F}_{2^{n}}$. A Boolean function on $\mathbf{F}_{2^{n}}$ is a function of the form $x \mapsto \operatorname{Tr}(P(x))$, where $P$ is any polynomial in $\mathbf{F}_{2^{n}}[x]$ and $\operatorname{Tr}$ is the trace function from $\mathbf{F}_{2^{n}}$ to $\mathbf{F}_{2}$. Such a function is said to be monomial when $P$ has only one term.

For any $k$ dividing $n$ and $n=u k$, we denote the trace function from $\mathbf{F}_{2^{n}}$ onto $\mathbf{F}_{2^{k}}$ as follows:

$$
T_{k}^{n}(b)=b+b^{2^{k}}+\cdots+b^{2^{k(u-1)}}, \quad b \in \mathbf{F}_{2^{n}} .
$$

Notation $\operatorname{Tr}$ is used for $k=1$.

\subsection{Boolean functions}

Let $f$ be any Boolean function on $\mathbf{F}_{2^{n}}$. The Hamming weight of $f$, denoted by $w t(f)$, is the number of $x \in \mathbf{F}_{2^{n}}$ such that $f(x)=1$. We denote by $\mathcal{F}(f)$ the following value related to the Walsh transform of $f$ :

$$
\mathcal{F}(f)=\sum_{x \in \mathbf{F}_{2^{n}}}(-1)^{f(x)}=2^{n}-2 w t(f) .
$$


The function $f$ is said to be balanced if and only if $\mathcal{F}(f)=0$ or, equivalently, $w t(f)=2^{n-1}$. The linear Boolean functions on $\mathbf{F}_{2^{n}}$ are the functions

$$
\varphi_{a}: x \mapsto \operatorname{Tr}(a x), \quad a \in \mathbf{F}_{2^{n}}
$$

The affine Boolean functions on $\mathbf{F}_{2^{n}}$ are $\varphi_{a}+c$ where $c \in \mathbf{F}_{2}$. The Walsh transform of $f$ is the mapping

$$
u \in \mathbf{F}_{2^{n}} \mapsto \mathcal{F}\left(f+\varphi_{u}\right)
$$

The Walsh spectrum of $f$ is the multiset

$$
\left\{\mathcal{F}\left(f+\varphi_{u}\right) \mid u \in \mathbf{F}_{2^{n}}\right\}
$$

Definition 1. For even $n$, a Boolean function $f$ on $\mathbf{F}_{2^{n}}$ is bent if and only if its Walsh transform takes the values $\pm 2^{n / 2}$ only.

More precisely, the Walsh spectrum of a bent function $f$ is:

\begin{tabular}{ll}
\hline $\mathcal{F}\left(f+\varphi_{u}\right)$ & Number of $u \in \mathbf{F}_{2^{n}}$ \\
\hline $2^{n / 2}$ & $2^{n-1}+(-1)^{f(0)} 2^{n / 2-1}$ \\
$-2^{n / 2}$ & $2^{n-1}-(-1)^{f(0)} 2^{n / 2-1}$ \\
\hline
\end{tabular}

The derivative of $f$ with respect to $b \in \mathbf{F}_{2^{n}}$, denoted by $D_{b} f$, is the Boolean function

$$
D_{b} f: x \mapsto f(x)+f(x+b) .
$$

The bent functions are exactly the ones which have all their derivatives $D_{b} f, b \neq 0$, balanced. Observe that if $f$ is a bent function and $L: \mathbf{F}_{2^{n}} \rightarrow \mathbf{F}_{2^{n}}$ is a affine permutation then $f \circ L$ is also bent. Also $f+l$ is bent for any affine function $l: \mathbf{F}_{2^{n}} \rightarrow \mathbf{F}_{2}$. The bent functions, which can be obtained from $f$ with such transformations, are called affinely equivalent to $f$.

\subsection{Quadratic Boolean functions}

Let $q$ be a power of 2 and $V$ be an $n$-dimensional vector space over $\mathbf{F}_{q}$. A map $Q: V \rightarrow \mathbf{F}_{q}$ is called a quadratic form on $V$ if

(a) $Q(c \mathbf{x})=c^{2} Q(\mathbf{x})$ for any $c \in \mathbf{F}_{q}$ and $\mathbf{x} \in V$,

(b) $B(\mathbf{x}, \mathbf{y}):=Q(\mathbf{x}+\mathbf{y})+Q(\mathbf{x})+Q(\mathbf{y})$ is bilinear on $V$.

The kernel $K$ of a quadratic form $Q$ is the subspace of $V$ defined by

$$
K=\{\mathbf{x} \in V: B(\mathbf{x}, \mathbf{y})=0 \text { for any } \mathbf{y} \in V\} .
$$


Given a basis $\left\{\gamma_{1}, \ldots, \gamma_{n}\right\}$ of $V$, let $\mathbf{x}=\sum_{i=1}^{n} x_{i} \gamma_{i}:=\left(x_{1}, \ldots, x_{n}\right)$, where $x_{i} \in \mathbf{F}_{q}$. Then using (a) and (b) we get

$$
Q(\mathbf{x})=Q\left(\sum_{i=1}^{n} x_{i} \gamma_{i}\right)=\sum_{i=1}^{n} Q\left(\gamma_{i}\right) x_{i}^{2}+\sum_{i<j} B\left(\gamma_{i}, \gamma_{j}\right) x_{i} x_{j}
$$

Hence, the quadratic form $Q$ can be expressed in the following form:

$$
Q(\mathbf{x})=\sum_{i \leqslant j} c_{i j} x_{i} x_{j}=\mathbf{x} \mathcal{C} \mathbf{x}^{t}
$$

where $\mathcal{C}$ is the upper triangular matrix with

$$
c_{i j}= \begin{cases}Q\left(\gamma_{i}\right) & \text { if } i=j \\ B\left(\gamma_{i}, \gamma_{j}\right) & \text { if } i<j\end{cases}
$$

Furthermore, (b) and (3) imply that $B(\mathbf{x}, \mathbf{y})=\mathbf{x} \mathcal{B} \mathbf{y}^{t}$, where $\mathcal{B}$ is the symmetric matrix $\mathcal{C}+\mathcal{C}^{t}$. The matrix $\mathcal{B}$ is alternating as well, i.e., $\mathbf{x} \mathcal{B} \mathbf{x}^{t}=0$. Indeed, $\mathbf{x} \mathcal{B} \mathbf{x}^{t}=B(\mathbf{x}, \mathbf{x})=0$. Note that the dimension $\mathbf{k}$ of the kernel $K$ is equal to the corank of the matrix $\mathcal{B}$

$$
\mathbf{k}=n-\operatorname{rank}(\mathcal{B})
$$

It is well known that the rank of an alternating matrix over any field is even [11, pp. 241, 242]. We collect the above information in the following proposition.

Proposition 1. Let $V$ be a vector space over a field $\mathbf{F}_{q}$ of characteristic 2 and $Q: V \rightarrow \mathbf{F}_{q}$ be a quadratic form. Then the dimension of $V$ and the dimension of the kernel of $Q$ have the same parity.

In this paper we are interested in the case where $V$ is an extension field $\mathbf{F}_{q^{n}}$ of $\mathbf{F}_{q}$. By counting it is easy to show that for any quadratic form $Q: \mathbf{F}_{q^{n}} \rightarrow \mathbf{F}_{q}$ there are unique $\delta_{i} \in \mathbf{F}_{q^{n}}, 0 \leqslant i \leqslant$ $\lfloor n / 2\rfloor$, such that

$$
Q(x)=T_{q}^{q^{n}}\left(\sum_{i=0}^{\lfloor n / 2\rfloor} \delta_{i} x^{q^{i}+1}\right)
$$

except when $n$ is even, in which case $\delta_{\lfloor n / 2\rfloor}$ is only unique modulo $\mathbf{F}_{q^{\lfloor n / 2\rfloor}}$ [14]. If $f: \mathbf{F}_{2^{n}} \rightarrow \mathbf{F}_{2}$ is a Boolean quadratic form, then its Walsh spectrum depends only on the dimension $\mathbf{k}$ of the kernel of $f$. More precisely, the Walsh spectrum of $f$ is:

\begin{tabular}{ll}
\hline $\mathcal{F}\left(f+\varphi_{u}\right)$ & Number of $u$ \\
\hline 0 & $2^{n}-2^{n-\mathbf{k}}$ \\
$2^{(n+\mathbf{k}) / 2}$ & $2^{n-\mathbf{k}-1}+(-1)^{f(0)} 2^{(n-\mathbf{k}-2) / 2}$ \\
$-2^{(n+\mathbf{k}) / 2}$ & $2^{n-\mathbf{k}-1}-(-1)^{f(0)} 2^{(n-\mathbf{k}-2) / 2}$ \\
\hline
\end{tabular}


Note that $f$ is bent if and only if $\mathbf{k}=0$. Assume that $f$ is a monomial function of the form $x \mapsto \operatorname{Tr}\left(a x^{2^{i}+1}\right)$, for some $i, 1 \leqslant i \leqslant\lfloor n / 2\rfloor$. Set $S_{i}=\left\{y^{2^{i}+1} \mid y \in \mathbf{F}_{2^{n}}^{*}\right\}$. Then the dimension of the kernel of $f$ is known to be

$$
\mathbf{k}= \begin{cases}\operatorname{gcd}(n, i) & \text { if } \operatorname{gcd}(n, i)=\operatorname{gcd}(n, 2 i), \\ \operatorname{gcd}(n, 2 i) & \text { if } 2 \operatorname{gcd}(n, i)=\operatorname{gcd}(n, 2 i) \text { and } a \in S_{i}, \\ 0 & \text { if } 2 \operatorname{gcd}(n, i)=\operatorname{gcd}(n, 2 i) \text { and } a \notin S_{i}\end{cases}
$$

These last properties are explained in [20, Chapter 15] (see Figs. 15.2 and 15.5) and [21]; see also [2, Proposition 4]. For more information on the Walsh transform of the monomial quadratic forms see [13, Appendix]. The next lemma can be directly obtained from the definitions.

Lemma 1. Let $f$ be any quadratic Boolean function. The kernel $K$ of $f$ is the subspace of those $b$ such that the derivative $D_{b} f$ is constant.

\subsection{The Maiorana-McFarland bent functions}

The Maiorana-McFarland class of bent functions was introduced in [22] and extensively studied by Dillon [12, pp. 90-95]. It is usually called the class $\mathcal{M}$ of bent functions. A bent function from $\mathcal{M}$ can be viewed as a concatenation of certain affine Boolean functions.

In the next lemma we define the subclass of $\mathcal{M}$ that we will consider later. The concatenation is made relatively to the cosets of $\mathbf{F}_{2^{t}}$, a structure which is particularly adapted to the monomial bent functions. The proof of this lemma is in fact a general proof for the class $\mathcal{M}$. We give a sketch of proof to define this subclass clearly.

Lemma 2. Let $n=2 t$ and $V=\mathbf{F}_{2^{t}}$. Denote by $W$ a subspace of the representatives of the cosets of $V$, that is $\mathbf{F}_{2^{n}}=\bigcup_{a \in W}(a+V)$. Let us consider a function $f$ on $\mathbf{F}_{2^{n}}$ defined by

$$
f:(y, a) \in V \times W \mapsto T_{1}^{t}(y \pi(a)+h(a)),
$$

where $\pi$ is a bijection from $W$ to $V$ and $h$ is any function from $W$ to $V$. Then, $f$ is a bent function which belongs to the class $\mathcal{M}$.

Sketch of proof. Fixing $a$ in (6), we get a function on $\mathbf{F}_{2^{t}}$, say $f_{a}$, which is affine relatively to $y$. Since $\pi$ is a permutation, only one $f_{a}$ is constant and all the others are balanced. Thus

$$
\mathcal{F}(f)=\sum_{a \in W} \sum_{y \in V}(-1)^{f(y, a)}=2^{t}(-1)^{T_{1}^{t}\left(h\left(a_{0}\right)\right)},
$$

where $\pi\left(a_{0}\right)=0$. Note that the functions $y \mapsto T_{1}^{t}(y \pi(a))$ form the set of all linear functions on $\mathbf{F}_{2^{t}}$. Consider any linear function $\ell$ on $\mathbf{F}_{2^{n}}$ and its restrictions $\ell_{a}$ on the cosets of $V$. If the kernel of $\ell$ contains $V$, then each $\ell_{a}$ is constant. Otherwise the $\ell_{a}$ are affine and there is one and only one $a$ such that $f_{a}+\ell_{a}$ is constant. Thus, $\mathcal{F}(f+\ell)$ and $\mathcal{F}(f)$ have the same absolute value.

Remark 1. Let $f$ be a bent function given by (6) and $g$ an affinely equivalent function given by

$$
g(x)=f \circ L(x)
$$


where $L$ is an affine permutation. Then $g$ is also in class $\mathcal{M}$. More precisely, it is a concatenation of affine functions on the subspace $L^{-1}(V)$. In particular, the composition $f \circ s_{\gamma}$ of the linear function

$$
s_{\gamma}: x \mapsto \gamma x
$$

and $f$ is a concatenation of affine functions on $\gamma^{-1} V$.

To introduce our method we consider in the next example the functions studied by Leander in [17]. We express such a function in a form close to form (6), which implies that a large part of these functions can be viewed as a concatenation of (not necessarily different) affine functions on the subfield.

Example 1. Let $n=4 r, r>1$. We consider the functions on $\mathbf{F}_{2^{n}}$ defined by

$$
g_{\lambda}: x \mapsto \operatorname{Tr}\left(\lambda x^{d}\right), \quad d=\left(2^{r}+1\right)^{2}, \lambda \in \mathbf{F}_{2^{2 r}}^{*}
$$

In [17] it is proved that these functions are bent if $\lambda \in \mathbf{F}_{4} \backslash\{0,1\}$ and $r$ is odd. We will show that $g_{\lambda}$, for any $r$ and for any $\lambda \in \mathbf{F}_{2^{2 r}}^{*}$, has a similar form to (6). The notation is the same as in the statement of Lemma 2: $V=\mathbf{F}_{2^{2 r}}$ and $W$ is a subspace of the representatives of the cosets of $V$ in $\mathbf{F}_{2^{n}}$. Then, for any $y \in V$ and $a \in W$, we compute:

$$
\begin{aligned}
g_{\lambda}(y, a)= & \operatorname{Tr}\left(\lambda(y+a)^{2^{2 r}+2^{r+1}+1}\right) \\
= & \operatorname{Tr}\left(\lambda y^{d}\right)+\operatorname{Tr}\left(\lambda\left(y^{1+2^{r+1}} a+y^{2^{r+1}+1} a^{2^{2 r}}+y^{2} a^{2^{r+1}}\right)\right) \\
& +\operatorname{Tr}\left(y\left(\lambda\left(a^{2^{2 r}+2^{r+1}}+a^{2^{r+1}+1}\right)+\lambda^{2^{r-1}} a^{2^{r-1}\left(2^{2 r}+1\right)}\right)\right) \\
& +\operatorname{Tr}\left(\lambda a^{2^{2 r}+2^{r+1}+1}\right)=A+B+C+D .
\end{aligned}
$$

We have $A=0$ since $\lambda$ and $y$ are in $\mathbf{F}_{2} 2 r$. Moreover,

$$
B=\operatorname{Tr}\left(\lambda y^{2^{r+1}+1}\left(a^{2^{2 r}}+a\right)+\lambda y^{2} a^{2^{r+1}}\right)=\operatorname{Tr}\left(\lambda y^{2} a^{2^{r+1}}\right),
$$

since $\lambda y^{2^{r+1}+1}\left(a^{2^{2 r}}+a\right) \in \mathbf{F}_{2^{2 r}}$. Thus we have that any function $g_{\lambda}$, defined by (7), is a concatenation of $2^{2 r}$ affine functions on $\mathbf{F}_{2^{2 r}}$. In order to specify the form of these functions, we compute the part which is linear relatively to $y$ :

$$
C=\operatorname{Tr}\left(y \lambda\left(a^{2^{2 r}+2^{r+1}}+a^{2^{r+1}+1}\right)\right),
$$

since $\lambda^{2^{r-1}} a^{2^{r-1}\left(2^{2 r}+1\right)} \in \mathbf{F}_{2^{2 r}}$. Finally

$$
g_{\lambda}(y, a)=T_{1}^{2 r}\left(y \pi(a)+\lambda T_{2 r}^{4 r}\left(a^{d}\right)\right),
$$

where $\pi$ is the mapping from $W$ to $V$ given by

$$
\pi(a)=\lambda\left(a+a^{2^{2 r}}\right)^{2^{r+1}+1}+\lambda^{2^{2 r-1}}\left(a+a^{2^{2 r}}\right)^{2^{r}} .
$$




\subsection{Definition of the functions $f_{\lambda}$}

Let $n=6 r, r>1$ and $\alpha$ be a primitive element of $\mathbf{F}_{2^{n}}$. Further let the functions $f_{\lambda}$ be defined by (1). Recall that $d=2^{2 r}+2^{r}+1$ and it holds

$$
2^{n}-1=\left(2^{3 r}-1\right)\left(2^{3 r}+1\right)=d\left(2^{2 r}-1\right)\left(2^{2 r}-2^{r}+1\right)
$$

If $\lambda=\gamma \theta^{d}$ for some $\gamma, \theta \in \mathbf{F}_{2^{n}}$, then $f_{\lambda}(x)=f_{\gamma}(\theta x)$, and we call $f_{\gamma}$ a shift of $f_{\lambda}$. A shift of $f_{\lambda}$ has the same Walsh spectrum as $f_{\lambda}$ does. Next we want to show that to obtain an information about Walsh spectrum of $f_{\lambda}$ it is enough to consider $\lambda \in \mathbf{F}_{2^{3 r}}$. Indeed, take the partition of the multiplicative group of $\mathbf{F}_{2^{n}}^{*}$ into the cosets of the subgroup $\left\langle\alpha^{d}\right\rangle$ generated with $\alpha^{d}$, i.e.

$$
\mathbf{F}_{2^{n}}^{*}=\bigcup_{i=0}^{d-1} \alpha^{i}\left\langle\alpha^{d}\right\rangle
$$

where $\left\langle\alpha^{d}\right\rangle=\left\{\alpha^{d \ell} \mid 0 \leqslant \ell<\frac{2^{n}-1}{d}\right\}$. Note that if two elements $\lambda$ and $\gamma$ belong to the same coset of $\left\langle\alpha^{d}\right\rangle$, then the functions $f_{\lambda}$ and $f_{\gamma}$ are shifts of each other. Finally, we observe that every coset of $\left\langle\alpha^{d}\right\rangle$ contains an element from the subfield $\mathbf{F}_{2^{3 r}}$. More precisely, for any $j, 0 \leqslant j \leqslant d-1$, $\alpha^{j\left(2^{3 r}+1\right)}$ belongs to one and only one $\alpha^{i}\left\langle\alpha^{d}\right\rangle$. Indeed, we have

$$
2^{3 r}+1=\left(2^{3 r}-1\right)+2 \equiv d\left(2^{r}-1\right)+2 \equiv 2 \quad(\bmod d) .
$$

Then $i=2 j$ if $0 \leqslant j \leqslant(d-1) / 2$ and $i=2 j-d$, otherwise.

Lemma 3. The subset of $\mathbf{F}_{2^{3 r}}$

$$
\left\{\alpha^{j\left(2^{3 r}+1\right)} \mid 0 \leqslant j \leqslant d-1\right\}
$$

is a set of the representatives of the cosets $\left\{\alpha^{i}\left\langle\alpha^{d}\right\rangle \mid 0 \leqslant i \leqslant d-1\right\}$ with

$$
j= \begin{cases}i / 2 & \text { if } i \text { is even } \\ (d+i) / 2 & \text { if } i \text { is odd }\end{cases}
$$

According to the previous lemma, to study the spectra of all the functions $f_{\lambda}$ defined by (1), it is sufficient to study the functions that we introduce below.

Definition 2. Let $n=6 r$ with $r>1$. Let us define the Boolean functions on $\mathbf{F}_{2^{n}}$ :

$$
f_{\lambda}(x)=\operatorname{Tr}\left(\lambda x^{d}\right), \quad \text { where }\left\{\begin{array}{l}
d=2^{2 r}+2^{r}+1, \\
\lambda=\alpha^{j\left(2^{3 r}+1\right)} \\
0 \leqslant j \leqslant d-1
\end{array}\right.
$$

Remark 2. When $r=1$, then $n=6$ and $d=7$. In this case, the bent functions $f_{\lambda}$ belong to the class of bent functions of the form: $x \mapsto \operatorname{Tr}\left(v x^{2^{t}-1}\right)$ (functions on $\mathbf{F}_{2^{n}}$ with $n=2 t$ ). These were studied by Dillon, as examples of the so-called $P S$ bent functions [12]. When $n=6$ we have 
$f_{\lambda}(x)=\operatorname{Tr}\left(\lambda x^{7}\right)$, with $\lambda \in \mathbf{F}_{8}$ and it is very easy to determine the bent functions. We know that $f_{\lambda}$ is bent if and only if the function on $\mathbf{F}_{8}$

$$
g_{\lambda}: x \mapsto T_{1}^{3}\left(x^{3}+\lambda x\right)
$$

is balanced (see [17, §II.B]). But it is well known that this cubic function is balanced if and only if $T_{1}^{3}(\lambda)=0$. We will see later that, in fact, this result holds when $r>1$.

\section{On the spectrum of $f_{\lambda}$}

In this section $f_{\lambda}$ is defined by (8). We denote by $\mathcal{G}$ the subgroup of $\mathbf{F}_{2^{n}}^{*}$ of order $2^{3 r}+1$. Since $\operatorname{gcd}\left(2^{3 r}+1,2^{3 r}-1\right)=1$, any $x \in \mathbf{F}_{2^{n}}^{*}$ has a unique representation:

$$
x=y z, \quad y \in \mathbf{F}_{2^{3 r}}^{*} \text { and } z \in \mathcal{G} .
$$

\subsection{The weight of $f_{\lambda}$}

Here we prove that $w t\left(f_{\lambda}\right)$ takes only three values (Theorem 1$)$. Recall that $\mathcal{F}\left(f_{\lambda}\right)=2^{n}-$ $2 w t\left(f_{\lambda}\right)$.

\section{Proposition 2. Let us define}

$$
L_{\lambda}=\left\{z \in \mathcal{G} \mid T_{r}^{6 r}\left(\lambda z^{d}\right) \neq 0\right\}
$$

Then the weight of $f_{\lambda}$ is $w t\left(f_{\lambda}\right)=2^{r-1} d \# L_{\lambda}$.

Proof. Using (9), we will express $w t\left(f_{\lambda}\right)$ as an integer sum on the pairs $(y, z)$. Note that $y^{d} \in$ $\mathbf{F}_{2^{r}}$, for $y \in \mathbf{F}_{2^{3 r}}$, since $2^{3 r}-1=\left(2^{r}-1\right) d$. So, it is clear that the application $y \mapsto y^{d}$ is $d$-to- 1 from $\mathbf{F}_{2^{3 r}}^{*}$ onto $\mathbf{F}_{2^{r}}^{*}$.

Now, we have:

$$
\begin{aligned}
w t\left(f_{\lambda}\right) & =\sum_{x \in \mathbf{F}_{2^{n}}} \operatorname{Tr}\left(\lambda x^{d}\right)=\sum_{z, y} \operatorname{Tr}\left(\lambda(z y)^{d}\right) \\
& =\sum_{z \in \mathcal{G}} \sum_{y \in \mathbf{F}_{2} 3 r} T_{1}^{r}\left(y^{d} T_{r}^{6 r}\left(\lambda z^{d}\right)\right) \\
& =\sum_{z \in \mathcal{G}} d \sum_{\rho \in \mathbf{F}_{2} r} T_{1}^{r}\left(\rho T_{r}^{6 r}\left(\lambda z^{d}\right)\right) \\
& =\# L_{\lambda} \times d \times 2^{r-1},
\end{aligned}
$$

since $\rho \mapsto T_{1}^{r}(\rho A)$ is linear on $\mathbf{F}_{2^{r}}$, for any $A \neq 0$.

Our next goal is to compute the cardinality of $L_{\lambda}$. Actually, we will compute the cardinality of $N_{\lambda}$, which is introduced in the next lemma. We denote by $\overline{L_{\lambda}}$ the set $\mathcal{G} \backslash L_{\lambda}$ :

$$
\overline{L_{\lambda}}=\left\{z \in \mathcal{G} \mid T_{r}^{6 r}\left(\lambda z^{d}\right)=0\right\}
$$


Lemma 4. Given a $\lambda \in \mathbf{F}_{2^{3 r}}$, set

$$
N_{\lambda}=\left\{y \in \mathbf{F}_{2^{3 r}}^{*} \mid T_{r}^{3 r}(\lambda y)=0 \text { and } T_{1}^{3 r}\left(\frac{1}{y}\right)=1\right\} .
$$

Then $\# \overline{L_{\lambda}}=2 \cdot \# N_{\lambda}+1$.

Proof. Note that $z^{2^{3 r}}=z^{-1}$ for any $z \in \mathcal{G}$. We have $z \in \overline{L_{\lambda}}$ if and only if $T_{r}^{6 r}\left(\lambda z^{d}\right)=0$. Since $\overline{L_{\lambda}}$ is contained in $\mathcal{G}$ and $\operatorname{gcd}\left(d, 2^{3 r}+1\right)=1$, the number of such $z$ is also obtained by taking as a condition $T_{r}^{6 r}(\lambda z)=0$. Thus we want to compute the number of $z$ satisfying

$$
T_{r}^{6 r}(\lambda z)=T_{r}^{3 r}\left(\lambda\left(z+z^{-1}\right)\right)=0,
$$

since $\lambda \in \mathbf{F}_{2^{3 r}}$. It is clear that $z$ satisfies the equality above if and only if $z^{-1}$ satisfies it too. On the other hand, it is well known that

$$
\left\{z+z^{-1} \mid z \in \mathcal{G} \backslash\{1\}\right\}=\left\{u \in \mathbf{F}_{2^{3 r}}^{*} \mid T_{1}^{3 r}\left(\frac{1}{u}\right)=1\right\}
$$

(see [16] for instance). Thus, computing $\# \overline{L_{\lambda}}$ is equivalent to computing the number of $u \in \mathbf{F}_{23 r}^{*}$ satisfying $T_{1}^{3 r}\left(u^{-1}\right)=1$ and $T_{r}^{3 r}(\lambda u)=0$, which is the cardinality of $N_{\lambda}$. Moreover, each such $u$ corresponds to a unique pair $\left(z, z^{-1}\right)$ and we add 1 , for $z=1$, to obtain the cardinality of $\overline{L_{\lambda}}$.

Theorem 1. Let the functions $f_{\lambda}$ (and $\lambda$ itself) be defined by (8). Let $G_{d}$ denote the subgroup of order $d$ of $\mathbf{F}_{2^{3 r}}^{*}$. Consider the solutions $v \in G_{d}$ of the equation:

$$
v^{2}+v \frac{T_{r}^{3 r}(\lambda)}{\lambda^{2^{r}}}+\frac{1}{\lambda^{2^{r}-1}}=0 .
$$

Then $\mathcal{F}\left(f_{\lambda}\right)=2^{n}-2^{r} d \# L_{\lambda}$ where $\# L_{\lambda}$ is computed as follows.

(a) If Eq. (11) has one and only one solution in $G_{d}$ then $\# L_{\lambda}=2^{2 r}\left(2^{r}-1\right)$; this holds if and only if $T_{r}^{3 r}(\lambda)=0$.

(b) If Eq. (11) has no solution in $G_{d}$ then $\# L_{\lambda}=2^{r}\left(2^{2 r}-2^{r}-1\right)$.

(c) Otherwise, Eq. (11) has two solutions in $G_{d}$ and $\# L_{\lambda}=2^{r}\left(2^{2 r}-2^{r}+1\right)$.

Consequently, we obtain:

\begin{tabular}{ll}
\hline Case & $\mathcal{F}\left(f_{\lambda}\right)$ \\
\hline (a) & $2^{3 r}$ \\
(b) & $2^{4 r}+2^{3 r+1}+2^{2 r}$ \\
(c) & $-\left(2^{4 r}+2^{2 r}\right)$ \\
\hline
\end{tabular}


Proof. Let $\beta$ be a primitive element of the subfield $\mathbf{F}_{2^{3 r}}$. According to Lemma 4, computing $\# L_{\lambda}$ is equivalent to computing $\# N_{\lambda}$. Since $2^{3 r}-1=\left(2^{r}-1\right) d$ we can express any $y \in \mathbf{F}_{2^{3 r}}^{*}$ as follows:

$$
y=u \beta^{i}, \quad u=\beta^{k d}, 0 \leqslant k \leqslant 2^{r}-2 \text { and } 0 \leqslant i \leqslant d-1 .
$$

Note that $u$ belongs to $\mathbf{F}_{2^{r}}^{*}$ while $\beta^{i}$ for $i \neq 0$ does not. Thus $T_{r}^{3 r}(\lambda y)=u T_{r}^{3 r}\left(\lambda \beta^{i}\right)$, which implies that (10) can be rewritten

$$
N_{\lambda}=\bigcup_{i=0}^{d-1}\left\{y \in \beta^{i} \mathbf{F}_{2^{r}}^{*} \mid T_{r}^{3 r}\left(\lambda \beta^{i}\right)=0 \text { and } T_{1}^{3 r}\left(\frac{1}{y}\right)=1\right\} .
$$

Let $I=\left\{0 \leqslant i \leqslant d-1 \mid T_{r}^{3 r}\left(\lambda \beta^{i}\right)=0\right\}$. The linear function $g: y \mapsto T_{r}^{3 r}(y)$ from $\mathbf{F}_{2^{3 r}}$ to $\mathbf{F}_{2^{r}}$ is surjective. So its kernel has dimension $2 r$. Moreover, $g(y)=0$ for $y \in \beta^{i} \mathbf{F}_{2^{r}}^{*}$ as soon as $g\left(\beta^{i}\right)=0$, and therefore

$$
\# I=\frac{2^{2 r}-1}{2^{r}-1}=2^{r}+1
$$

Hence, with $y=u \beta^{i}$,

$$
\begin{aligned}
\# N_{\lambda} & =\sum_{i \in I} \#\left\{y \in \beta^{i} \mathbf{F}_{2^{r}}^{*} \mid T_{1}^{3 r}\left(\frac{1}{y}\right)=1\right\} \\
& =\sum_{i \in I} \#\left\{u \in \mathbf{F}_{2^{r}}^{*} \mid T_{1}^{r}\left(\frac{1}{u} T_{r}^{3 r}\left(\beta^{-i}\right)\right)=1\right\} \\
& =2^{r-1} \times \#\left\{i \in I \mid T_{r}^{3 r}\left(\beta^{-i}\right) \neq 0\right\} \\
& =2^{r-1}\left(2^{r}+1-\#\left\{i \in I \mid T_{r}^{3 r}\left(\beta^{-i}\right)=0\right\}\right) .
\end{aligned}
$$

Hence in order to find $\# N_{\lambda}$, we have to compute the number of $i$ such that $i \in I$ and $T_{r}^{3 r}\left(\beta^{-i}\right)=0$. Setting $w=\beta^{i}, 0 \leqslant i \leqslant d-1$, we have to solve the system:

$$
\left\{\begin{array}{l}
\lambda w+(\lambda w)^{2^{r}}+(\lambda w)^{2^{2 r}}=0 \\
\frac{1}{w}+\frac{1}{w^{2^{r}}}+\frac{1}{w^{2^{2 r}}}=0
\end{array}\right.
$$

Note that $w=1$ (i.e., $i=0$ ) is not a solution of (13). So we have $w^{2^{r}-1} \neq 1$, which allows us to express $w^{2^{2 r}}$ from the second equation:

$$
w^{2^{2 r}}=\frac{w^{2^{r}}}{w^{2^{r}-1}+1}
$$

that we substitute in the first equation. We can multiply this first equation by $w^{2^{r}-1}+1$ and we get:

$$
\lambda\left(w^{2^{r}}+w\right)+\lambda^{2^{r}}\left(w^{2^{r+1}-1}+w^{2^{r}}\right)+\lambda^{2^{2 r}} w^{2^{r}}=0
$$


which gives, with $\delta=T_{r}^{3 r}(\lambda)$,

$$
\delta w^{2^{r}}+\lambda w+\lambda^{2^{r}} w^{2^{r+1}-1}=0 .
$$

Dividing the previous equation by $\lambda^{2^{r}} w$, we finally get:

$$
w^{2\left(2^{r}-1\right)}+w^{2^{r}-1} \frac{\delta}{\lambda^{2^{r}}}+\frac{1}{\lambda^{2^{r}-1}}=0 .
$$

Setting $v=w^{2^{r}-1}$, we get Eq. (11). Note that, by definition, $v$ describes the subgroup $G_{d}$ of $\mathbf{F}_{2^{3 r}}^{*}$ of order $d$. Also, observe that because of definition of $w$, the correspondence $w \mapsto v$ is a bijection.

Equation (11) is an equation of degree 2 which has either 0 or 1 or 2 solutions in $\mathbf{F}_{23 r}^{*}$. If it has 2 solutions, $v_{1}$ and $v_{2}$, then $v_{1} \in G_{d}$ implies $v_{2} \in G_{d}$ since $v_{1} v_{2}=1 / \lambda^{2^{r}-1}$. So (11) has one and only one solution in $G_{d}$ if and only if $\delta=0$. Using (12) we get $\# N_{\lambda}=2^{r-1} 2^{r}$; moreover, by Lemma 4, we have

$$
\# L_{\lambda}=2^{3 r}+1-2^{2 r}-1=2^{r}\left(2^{2 r}-2^{r}\right) .
$$

In the same way, when (11) has no solution in $G_{d}$ we get $\# N_{\lambda}=2^{r-1}\left(2^{r}+1\right)$ and then $\# L_{\lambda}=2^{r}\left(2^{2 r}-2^{r}-1\right)$. If (11) has 2 solutions in $G_{d}$ then $\# N_{\lambda}=2^{r-1}\left(2^{r}-1\right)$ and $\# L_{\lambda}=$ $2^{r}\left(2^{2 r}-2^{r}+1\right)$, completing the proof of the cases (a)-(c). Then we are able to compute $\mathcal{F}\left(f_{\lambda}\right)$, using Proposition 2 and $\mathcal{F}\left(f_{\lambda}\right)=2^{n}-2 w t\left(f_{\lambda}\right)$.

Remark 3. We call the case (a) the bent case since $f_{\lambda}$ could be bent in this case only. Clearly there are $\lambda$ such that $\delta=T_{r}^{3 r}(\lambda)=0$. Moreover, our numerical results show that there are $\lambda$ such that case (b) (respectively case (c)) holds. Note that Eq. (11) has no solution in $\mathbf{F}_{2^{3 r}}$ if and only if

$$
T_{1}^{3 r}\left(\frac{\lambda^{2^{r}+1}}{\delta^{2}}\right)=T_{1}^{r}\left(\frac{1}{\delta^{2}} T_{r}^{3 r}\left(\lambda^{2^{r}+1}\right)\right)=1 .
$$

\subsection{Another expression of $f_{\lambda}$}

In this subsection we want to express any function $f_{\lambda}$ by means of its restrictions on the (additive) cosets of $\mathbf{F}_{2^{3 r}}$. We proceed as in Section 2.3. Set $V=\mathbf{F}_{2^{3 r}}$ and let $W$ be a subspace of $\mathbf{F}_{2^{n}}$ which is a set of the representatives of the cosets of $V$. Thus, for any $x \in \mathbf{F}_{2^{n}}$ there is a unique pair $(y, a) \in V \times W$ such that $x=y+a$. Then, we define

$$
f_{\lambda}(y, a)=f_{\lambda}(y+a)=\operatorname{Tr}\left(\lambda(y+a)^{d}\right) .
$$

Theorem 2. Let $\delta=T_{r}^{3 r}(\lambda)$. Define the function $\pi$ from $W$ to $V$ :

$$
\pi(a)=\lambda^{2^{2 r}}\left(a+a^{2^{3 r}}\right)^{2^{2 r}+2^{r}}+\delta T_{3 r}^{6 r}\left(a^{2^{2 r}+2^{r}}\right) .
$$

Then, for any $(y, a) \in V \times W$ :

$$
f_{\lambda}(y, a)=T_{1}^{3 r}\left(y^{2^{r}+1} \delta\left(a+a^{2^{3 r}}\right)^{2^{2 r}}+y \pi(a)+\lambda\left(a^{d}+a^{2^{3 r}}\right)\right) .
$$


Proof. We compute $f_{\lambda}(y, a)$, which is defined by (14):

$$
\begin{aligned}
f_{\lambda}(y, a)= & \operatorname{Tr}\left(\lambda(y+a)^{d}\right) \\
= & \operatorname{Tr}\left(\lambda y^{d}\right)+\operatorname{Tr}\left(\lambda\left(a^{2^{2 r}} y^{2^{r}+1}+a^{2^{r}} y^{2^{2 r}+1}+a y^{2^{r}\left(2^{r}+1\right)}\right)\right) \\
& +\operatorname{Tr}\left(\lambda\left(y^{2^{2 r}} a^{2^{r}+1}+y^{2^{r}} a^{2^{2 r}+1}+y a^{2^{r}\left(2^{r}+1\right)}+a^{d}\right)\right) \\
= & A+B+C .
\end{aligned}
$$

First $A=\operatorname{Tr}\left(\lambda y^{d}\right)=0$ since $\lambda$ and $y$ are in $\mathbf{F}_{2^{3 r}}$. Now, using the properties of the trace function, we have

$$
\begin{aligned}
B & =\operatorname{Tr}\left(y^{2^{r}+1}\left(\lambda a^{2^{2 r}}+\lambda^{2^{r}} a^{2^{2 r}}+\lambda^{2^{2 r}} a^{2^{2 r}}\right)\right) \\
& =\operatorname{Tr}\left(y^{2^{r}+1} a^{2^{2 r}}\left(\lambda+\lambda^{2^{r}}+\lambda^{2^{2 r}}\right)\right) \\
& =T_{1}^{3 r}\left(y^{2^{r}+1} \delta\left(a+a^{2^{3 r}}\right)^{2^{2 r}}\right) .
\end{aligned}
$$

Finally the part which is affine relatively to $y$ is:

$$
\begin{aligned}
C & =\operatorname{Tr}\left(y\left(\lambda a^{2^{r}\left(2^{r}+1\right)}+\lambda^{2^{r}} a^{2^{r}\left(2^{r}+1\right)}+\lambda^{2^{2 r}} a^{2^{2 r}\left(2^{2 r}+1\right)}\right)+\lambda a^{d}\right) \\
& =T_{1}^{3 r}\left(y T_{3 r}^{6 r}(D)+\lambda T_{3 r}^{6 r}\left(a^{d}\right)\right),
\end{aligned}
$$

where

$$
\begin{aligned}
T_{3 r}^{6 r}(D) & =\left(\lambda^{2^{2 r}}+\delta\right) T_{3 r}^{6 r}\left(a^{2^{2 r}+2^{r}}\right)+\lambda^{2^{2 r}} T_{3 r}^{6 r}\left(a^{2^{4 r}+2^{2 r}}\right) \\
& =\lambda^{2^{2 r}} T_{3 r}^{6 r}\left(a^{2^{2 r}}\left(a+a^{2^{3 r}}\right) 2^{2^{r}}\right)+\delta T_{3 r}^{6 r}\left(a^{2^{2 r}+2^{r}}\right) \\
& =\lambda^{2^{2 r}}\left(a+a^{2^{3 r}}\right)^{2^{2 r}+2^{r}}+\delta T_{3 r}^{6 r}\left(a^{2^{2 r}+2^{r}}\right)
\end{aligned}
$$

which is exactly $\pi(a)$, completing the proof of (16).

\subsection{The bent functions}

Now we use Lemma 2 to characterize those $\lambda$ such that $f_{\lambda}$ is bent. A part of these bent functions form a subspace of the Boolean functions of degree 3 .

Theorem 3. The function $f_{\lambda}$, defined by (8), is bent if and only if $T_{r}^{3 r}(\lambda)=0$. There are $2^{r}+1$ such bent functions. In general, if $\lambda$ runs through $\mathbf{F}_{2^{n}}^{*}$, then there are

$$
\left(2^{2 r}-1\right)\left(2^{3 r}+1\right)
$$

bent functions $f_{\lambda}$, defined in (1). All these bent functions belong to the class $\mathcal{M}$. Moreover, the set

$$
\mathcal{B}=\left\{f_{\lambda} \mid \lambda \in \mathbf{F}_{2^{3 r}}, T_{r}^{3 r}(\lambda)=0\right\}
$$


where $f_{0}$ is the null function, is a subspace of the vector space of Boolean functions on $\mathbf{F}_{2^{n}}$ over $\mathbf{F}_{2}$. Its dimension is $2 r$ and any function $f_{\lambda} \in \mathcal{B}^{*}$ is a cubic bent function.

Proof. First, if $\delta \neq 0$ then $f_{\lambda}$ cannot be bent, since $\mathcal{F}\left(f_{\lambda}\right) \notin\left\{ \pm 2^{3 r}\right\}$. This was proved by Theorem 1. So, we consider the functions $f_{\lambda}$ expressed by (16) with $\delta=0$. Notation is as in Theorem 2. Then we get:

$$
f_{\lambda}(y, a)=T_{1}^{3 r}\left(y \pi(a)+\lambda\left(a^{d}+a^{2^{3 r}}\right)\right)
$$

with $\pi(a)=\lambda^{2^{2 r}}\left(a+a^{2^{3 r}}\right)^{2^{2 r}+2^{r}}$. The linear function $a \mapsto a+a^{2^{3 r}}$ is a bijection from $W$ to $V$, since its kernel is $W \cap V=\{0\}$. We conclude that $\pi$ is a bijection from $W$ to $V$ because $2^{r}+1$ and $2^{3 r}-1$ are coprime. According to Lemma 2, the functions expressed by (17) are bent functions belonging to the class $\mathcal{M}$. Now, set

$$
S=\left\{\lambda=\alpha^{\ell\left(2^{3 r}+1\right)} \mid 0 \leqslant \ell \leqslant d-1, T_{r}^{3 r}(\lambda)=0\right\} .
$$

Note that $S$ is the set of those $\lambda$ corresponding to bent functions defined by (8). The application $y \mapsto T_{r}^{3 r}(y)$, from $V$ to $\mathbf{F}_{2^{r}}$, has a kernel of dimension $2 r$. Moreover, for any $\lambda \in S$ we have $T_{r}^{3 r}(\lambda u)=0$ for any $u \in \mathbf{F}_{2^{r}}^{*}$. Then $\# S=\left(2^{2 r}-1\right) /\left(2^{r}-1\right)=2^{r}+1$, implying that there are $2^{r}+1$ bent functions defined by $(8)$, each of them having $\left(2^{n}-1\right) / d$ shifts. Thus, we get

$$
\left(2^{r}+1\right)\left(2^{3 r}+1\right)\left(2^{r}-1\right)=\left(2^{2 r}-1\right)\left(2^{3 r}+1\right)
$$

bent functions $f_{\lambda}$, when $\lambda$ runs through $\mathbf{F}_{2^{n}}^{*}$ (see Section 2.4). Moreover, as it was explained in Remark 1 these shifts are also elements of the class $\mathcal{M}$.

Let $\lambda \in S$. Consider the shifts $f_{u \lambda}$ of $f_{\lambda}$ such that $u \lambda \in \mathbf{F}_{2^{3 r}}$. This holds for $u \in \mathbf{F}_{2^{r}}^{*}$ only, since we must have $u=v^{d}$ for some $v$ in $\mathbf{F}_{2^{3 r}}$. So we have $2^{r}-1$ such shifts. Since $T_{r}^{3 r}(u \lambda)=0$, we have proved that $\mathcal{B}$ contains $2^{2 r}-1$ bent functions. $\mathcal{B}$ is a subspace because of the linearity of the trace function: if $f_{\lambda}$ and $f_{\mu}$ are in $\mathcal{B}$ then $f_{\lambda+\mu} \in \mathcal{B}$. All functions $f_{\lambda}$ are of degree 3 , completing the proof.

To illustrate the previous theorem, we compute the number of bent functions for $r=2$.

Example 2. Let $r=2$; so $n=12$ and $d=21$. At first let us look more closely on the numerical results given in Table 1 . They show that $f_{\alpha^{i}}$ is bent for $i \in\{7,9\}$, where $\alpha$ is a primitive element of $\mathbf{F}_{2^{12}}$. Then we get five cosets $\alpha^{i}\left\langle\alpha^{21}\right\rangle$ whose elements define bent functions. Indeed $i$ is a representative of its 2-cyclotomic coset modulo 21: 7 is the representative of $\{7,14\}$ and 9 is the representative of $\{9,15,18\}$. So, we get at all $975=5 \times 195$ bent functions.

Now, using Lemma 3 and the previous calculation, we get five bent functions $f_{\lambda}$ with $\lambda \in \mathbf{F}_{2^{6}}$ :

$$
\lambda=\alpha^{65 j}, \quad j \in J, J=\{7,14,9,15,18\} .
$$

And, using Theorem 3, there are $975=15 \times 65$ many bent $f_{\lambda}$ if $\lambda \in \mathbf{F}_{2^{12}}$. The subspace of bent functions of dimension 4 is obtained by taking all elements $\lambda$ in $\alpha^{65 j}\left\langle\alpha^{21}\right\rangle, j \in J$, which belong also to the subfield $\mathbf{F}_{2^{6}}$, that is

$$
\lambda=\alpha^{65 \ell}, \quad \ell=j+k d, j \in J \text { and } 0 \leqslant k \leqslant 2 .
$$


Table 1

Walsh spectra of $f_{\alpha^{i}}$ over $\mathbf{F}_{2^{12}}$ where $\alpha$ is a root of the primitive polynomial $x^{12}+x^{6}+x^{4}+x+1$. It corresponds to the case $r=2, n=12$ and $d=21$. The spectra of the functions $x \mapsto \operatorname{Tr}\left(\alpha^{i} x^{21}\right)$, where $i \in I$ with $I=\{0,1,3,5,7,9\}$, are presented. The set $I$ is a set of representatives of the 2-cyclotomic cosets modulo 21 . In this table, a spectrum is presented as a list: value [number] value $[$ number $]$.... The set of all bent functions is described in Example 2

\begin{tabular}{lll}
\hline$i$ & Weight of $f$ & Spectra \\
\hline 0 & $2^{11}+136$ & $112[546] 48[1092]-16[1365]-80[1092]-272[1]$ \\
1 & $2^{11}+136$ & $112[546] 48[1092]-16[1365]-80[1092]-272[1]$ \\
3 & $2^{11}+136$ & $112[546] 48[1092]-16[1365]-80[1092]-272[1]$ \\
5 & $2^{11}-200$ & $400[1] 144[441] 80[84] 16[1764]-48[1764]-240[42]$ \\
7 & $2^{11}-32$ & $64[2080]-64[2016]$ \\
9 & $2^{11}-32$ & $64[2080]-64[2016]$ \\
\hline
\end{tabular}

We get 15 such bent functions.

\subsection{Functions which are not bent}

In this section, we consider the functions $f_{\lambda}$ which are not bent. We obtain the divisibility of the Walsh transform of such $f_{\lambda}$ from Theorems 1 and 2 .

Corollary 1. Assume that $T_{r}^{3 r}(\lambda) \neq 0$, i.e. $f_{\lambda}$ is not bent. Set $s=2 r$. Then for all $b \in \mathbf{F}_{2^{n}}$

$$
\mathcal{F}\left(f_{\lambda}+\varphi_{b}\right) \equiv 0 \quad\left(\bmod 2^{s}\right)
$$

Moreover, this does not hold for $s>2 r$.

Proof. Notation is as in Theorem 2. When $f_{\lambda}$ is not bent, we have seen that it can be expressed as a concatenation of quadratic functions of the form $T_{1}^{3 r}\left(v y^{2^{r}+1}+c y+c^{\prime}\right)$. This property holds for $f_{\lambda}+\varphi_{b}$ for any $b$. Indeed

$$
\begin{aligned}
\left(f_{\lambda}+\varphi_{b}\right)(y+a) & =f_{\lambda}(y+a)+\operatorname{Tr}(b(y+a)) \\
& =T_{1}^{3 r}\left(v y^{2^{r}+1}+y\left(c+T_{3 r}^{6 r}(b)\right)+c^{\prime}+T_{3 r}^{6 r}(b a)\right)
\end{aligned}
$$

and we have

$$
\mathcal{F}\left(f_{\lambda}+\varphi_{b}\right)=\sum_{a} \sum_{y}(-1)^{\left(f_{\lambda}+\varphi_{b}\right)(y, a)}
$$

Any quadratic function of the form $y \mapsto T_{1}^{3 r}\left(v y^{2^{r}+1}\right)$ on $\mathbf{F}_{2^{3 r}}$ is such that the values of its Walsh transform are $\left\{0, \pm 2^{2 r}\right\}$ when $v \neq 0$. This is because the dimension $\mathbf{k}$ of its kernel equals $r$ (see Section 2.2). We can conclude that $\mathcal{F}\left(f_{\lambda}+\varphi_{b}\right)$ is divisible by $2^{2 r}$. By Theorem 1 , we know that $\mathcal{F}\left(f_{\lambda}\right)$ is divisible by $2^{2 r}$ and not divisible by $2^{2 r+1}$, completing the proof.

Concerning the non-bent spectra, our numerical results lead to several conjectures that we list below. To illustrate our purpose, we present the case $r=2$ in Table 1. 
Conjecture 1. There are three different spectra only, one "bent" and two "non-bent," for the functions $f_{\lambda}$. These three spectra correspond to the three cases listed in Theorem 1.

For any non-bent function $f_{\lambda}$, the value $\mathcal{F}\left(f_{\lambda}\right)$ appears only once and the value 0 never appears.

\section{Some quadratic functions: the derivatives}

The derivative of $f_{\lambda}\left(\lambda \in \mathbf{F}_{2^{n}}^{*}\right)$ with respect to $a \in \mathbf{F}_{2^{n}}^{*}$ is the function

$$
D_{a} f_{\lambda}(x)=\operatorname{Tr}\left(\lambda x^{d}\right)+\operatorname{Tr}\left(\lambda(x+a)^{d}\right) .
$$

Our purpose, in this section, is to study some properties of these specific quadratic functions. Firstly, we are interested in the Walsh spectra of these functions. To obtain the Walsh spectra of the functions $D_{a} f_{\lambda}$ it is enough to consider the spectra of the functions $D_{1} f_{\mu}$ with $\mu=\lambda a^{d}$. Indeed,

$$
\begin{aligned}
D_{a} f_{\lambda}(x) & =\operatorname{Tr}\left(\lambda x^{d}\right)+\operatorname{Tr}\left(\lambda(x+a)^{d}\right) \\
& =\operatorname{Tr}\left(\lambda a^{d}\left(a^{-1} x\right)^{d}\right)+\operatorname{Tr}\left(\lambda a^{d}\left(a^{-1} x+1\right)^{d}\right) \\
& =D_{1} f_{\lambda a^{d}}\left(a^{-1} x\right) .
\end{aligned}
$$

The following proposition gives more information about $D_{a} f_{\lambda}$.

Proposition 3. Let $a \in \mathbf{F}_{2^{n}}^{*}$ and $\mu=\lambda a^{d}$. Then $D_{1} f_{\mu}$ is the function $g_{a, \lambda}$ given by

$$
g_{a, \lambda}(x)=\operatorname{Tr}\left(\mu x^{2^{2 r}+1}+A x^{2^{r}+1}+B x+\mu\right),
$$

with

$$
A=\mu+\mu^{2^{5 r}}, \quad B=\mu+\mu^{2^{4 r}}+\mu^{2^{5 r}} .
$$

Consequently,

$$
D_{a} f_{\lambda}(x)=g_{a, \lambda}\left(a^{-1} x\right)
$$

Proof. According to the previous remark, it is sufficient to compute $D_{1} f_{\lambda a^{d}}$ (denoted by $g_{a, \lambda}$ ):

$$
\begin{aligned}
g_{a, \lambda}(x) & =\operatorname{Tr}\left(\mu\left[x^{2^{2 r}+2^{r}}+x^{2^{2 r}+1}+x^{2^{r}+1}+x^{2^{2 r}}+x^{2^{r}}+x+1\right]\right) \\
& =\operatorname{Tr}\left(\mu x^{2^{2 r}+1}+\left(\mu+\mu^{2^{5 r}}\right) x^{2^{r}+1}+\left(\mu+\mu^{2^{4 r}}+\mu^{2^{5 r}}\right) x+\mu\right) .
\end{aligned}
$$




\subsection{The kernels of derivatives}

The Walsh spectrum of the function $g_{a, \lambda}$ is equal to the one of the following functions:

$$
h_{\mu}(x)=\operatorname{Tr}\left(\mu x^{2^{2 r}+1}+\left(\mu+\mu^{2^{5 r}}\right) x^{2^{r}+1}\right), \quad \mu=\lambda a^{d},
$$

for any $a$ and any $\lambda$ in $\mathbf{F}_{2^{n}}^{*}$. Note that $h_{\mu}$ is a quadratic form from $\mathbf{F}_{2^{n}}$ into $\mathbf{F}_{2}$. Thus using the results of Section 2.2, the Walsh spectrum of $h_{\mu}$ is completely defined as soon as the dimension of its kernel is known. Our next goal is to describe this kernel.

Lemma 5. Let $K(\mu)$ be the kernel of the quadratic form $h_{\mu}$ and $A=\mu+\mu^{2^{5 r}}$. Then $K(\mu)$ is the subspace of the roots of $P \in \mathbf{F}_{2^{n}}[x]$ given by

$$
P(x)=\mu x^{2^{2 r}}+A x^{2^{r}}+(\mu x)^{2^{4 r}}+(A x)^{2^{5 r}} .
$$

Proof. We compute the derivatives of $h_{\mu}$ with respect to any $b \in \mathbf{F}_{2^{n}}^{*}$ :

$$
\begin{aligned}
D_{b} h_{\mu}(x) & =\operatorname{Tr}\left(\mu\left(x^{2^{2 r}} b+b^{2^{2 r}} x\right)+A\left(x^{2^{r}} b+b^{2^{r}} x\right)+\mu b^{2^{2 r}+1}+A b^{2^{r}+1}\right) \\
& =\operatorname{Tr}\left(x\left(\mu b^{2^{2 r}}+A b^{2^{r}}+(\mu b)^{2^{4 r}}+(A b)^{2^{5 r}}\right)\right)+h_{\mu}(b) .
\end{aligned}
$$

According to Lemma 1, we get for any $\mu \in \mathbf{F}_{2^{n}}^{*}$

$$
K(\mu)=\left\{b \in \mathbf{F}_{2^{n}} \mid \mu b^{2^{2 r}}+A b^{2^{r}}+(\mu b)^{2^{4 r}}+(A b)^{2^{5 r}}=0\right\} .
$$

Recall that a polynomial of the form $\sum_{i=0}^{m-1} a_{i} x^{q^{i}}$ with coefficients in an extension field $\mathbf{F}_{q^{m}}$ of $\mathbf{F}_{q}$ is called a q-polynomial over $\mathbf{F}_{q^{m}}$ [19, p.107]. If $\mathbf{F}_{q^{m}}$ is considered as a vector space over $\mathbf{F}_{q}$, then $q$-polynomials are the linear maps of this vector space. Hence we can speak from the kernel of a $q$-polynomial. Clearly, the kernel and the image set of a $q$-polynomial are subspaces of $\mathbf{F}_{q^{m}}$ over $\mathbf{F}_{q}$. In particular, these sets have cardinality $q^{k}$ for some $k$. The polynomial $P(x)$ considered here is a $2^{r}$-polynomial. As a consequence, the dimension of the kernel of $P(x)$ (i.e., the dimension of any $K(\mu))$ equals $k r$ for some $k$. On the other hand, $K(\mu)$ has at most $2^{4 r}$ elements because $P(x)$ can be written as $\left(P^{\prime}(x)\right)^{2^{r}}$ with deg $P^{\prime}=2^{4 r}$.

Consider now the quadratic form from $\mathbf{F}_{q^{6}}$ to $\mathbf{F}_{q}\left(q=2^{r}\right)$ :

$$
H_{\mu}(x)=T_{r}^{6 r}\left(\mu x^{2^{2 r}+1}+A x^{2^{r}+1}\right)
$$

The set of roots of $P(x)$ is also the kernel $\mathcal{K}$ of $H_{\mu}$. Indeed, $\mathcal{K}$ is the set of those $b$ such that $B(x)=0$ for all $x$ with

$$
B(x)=H_{\mu}(x)+H_{\mu}(b)+H_{\mu}(x+b)
$$

(see Section 2.2). Since $D_{b} h_{\mu}(x)=T_{1}^{r}(B(x)$ ), we get

$$
B(x)=T_{r}^{6 r}(P(b) x)
$$


(see the proof of Lemma 5). Thus, the kernel $\mathcal{K}$ of $H_{\mu}$ is equal to $K(\mu)$. By Proposition 1 , the dimension of $\mathcal{K}$ over $\mathbf{F}_{2^{r}}$ must have the same parity as 6 , so it is even. We conclude that the dimension of $\mathcal{K}$ over $\mathbf{F}_{2^{r}}$ is either 2 or 4 , implying that the one of $K(\mu)$ over $\mathbf{F}_{2}$ is either $2 r$ or $4 r$.

Proposition 4. The kernel $K(\mu)$ of the quadratic function $h_{\mu}$, defined by (19), has dimension either $2 r$ or $4 r$.

\subsection{The spectrum of $h_{\mu}$}

In this subsection we determine for which $\mu$ the dimension of $K(\mu)$ is $2 r$ and for which it is $4 r$, studying the kernel of $P(x)$. First we prove two lemmas. The first lemma can be easily generalized to any finite field.

Lemma 6. Let $q=2^{r}, n=r m$ and $U(x)$ be any q-polynomial over $\mathbf{F}_{2^{n}}$. Set $\operatorname{Im} U=$ $\left\{U(x) \mid x \in \mathbf{F}_{2^{n}}\right\}$

$$
V=\left\{x \in \mathbf{F}_{2^{n}} \mid U(x)+U(x)^{2^{r}}=0\right\} \quad \text { and } \quad W=\left\{x \in \mathbf{F}_{2^{n}} \mid U(x)=0\right\} .
$$

Then, $\operatorname{Im} U \cap \mathbf{F}_{2^{r}}$ equals either $\mathbf{F}_{2^{r}}$ or $\{0\}$. Furthermore, $\operatorname{dim} V$ is equal to

$$
\begin{cases}\operatorname{dim} W & \text { if } \operatorname{Im} U \cap \mathbf{F}_{2^{r}}=\{0\}, \\ \operatorname{dim} W+r & \text { if } \operatorname{Im} U \cap \mathbf{F}_{2^{r}}=\mathbf{F}_{2^{r}} .\end{cases}
$$

Proof. Suppose that there exist $\xi \in F_{2^{r}}^{*}$ and $x_{0} \in \mathbf{F}_{2^{n}}$ such that $U\left(x_{0}\right)=\xi$. Then for any $\delta \in \mathbf{F}_{2^{r}}$ it holds

$$
U\left(\delta \xi^{-1} x_{0}\right)=\delta \xi^{-1} U\left(x_{0}\right)=\delta
$$

proving the first statement. Recall that $U(x)+U(x)^{2^{r}}=0$ if and only if $U(x) \in \mathbf{F}_{2^{r}}$. To complete the proof note that every element in $\operatorname{Im} U$ has $2^{u}$ many preimages, where $u=\operatorname{dim} W$.

We again consider $K(\mu)$ and $P(x)$, as defined by Lemma 5 .

Lemma 7. For any $\mu$, we have $P(x)=L\left(x^{2^{r}}+x\right)$ with

$$
L(x)=\mu x^{2^{r}}+(\mu x)^{2^{4 r}}+\mu^{2^{5 r}}\left(x^{2^{5 r}}+x\right) .
$$

In particular, $K(\mu)$ contains $\mathbf{F}_{2^{r}}$. Furthermore, denoting by $I$ be the image set of the mapping $x \mapsto x^{2^{r}}+x, x \in \mathbf{F}_{2^{n}}$, we have

$$
\operatorname{dim} K(\mu)=\operatorname{dim}\{x \in I \mid L(x)=0\}+r
$$

Proof. We have: 


$$
\begin{aligned}
P(x) & =\mu x^{2^{2 r}}+\left(\mu+\mu^{2^{5 r}}\right) x^{2^{r}}+\mu^{2^{4 r}} x^{2^{4 r}}+\left(\mu^{2^{5 r}}+\mu^{2^{4 r}}\right) x^{2^{5 r}} \\
& =\mu\left(x+x^{2^{r}}\right)^{2^{r}}+\mu^{2^{4 r}}\left(x+x^{2^{r}}\right)^{2^{4 r}}+\mu^{2^{5 r}}\left(x+x^{2^{4 r}}\right)^{2^{r}} \\
& =L\left(x+x^{2^{r}}\right)
\end{aligned}
$$

with

$$
x+x^{2^{4 r}}=\left(x+x^{2^{r}}\right)^{2^{4 r}}+\left(x+x^{2^{r}}\right)^{2^{5 r}} .
$$

Since $K(\mu)$ is the kernel of $P$, equality (21) is directly obtained. We use that every element from $I$ has $2^{r}$ preimages.

Proposition 4 and Lemma 7 imply that $P(x)$ has always some roots which do not belong to $\mathbf{F}_{2^{r}}$. Actually, we have to find the nonzero $X$ such that

$$
L(X)=0, \quad X=x^{2^{r}}+x, x \in \mathbf{F}_{2^{n}},
$$

where $L$ is defined by (20). Note that $X=x^{2^{r}}+x$ if and only if $T_{r}^{6 r}(X)=0$.

Lemma 8. Let $\sigma=\mu^{2^{r}+2^{4 r}}+\mu^{2^{r}+2^{3 r}}+\mu^{2^{2 r}+2^{4 r}}$. The polynomial $L$ is given by (20). Then we have:

(a) Assume $\sigma \neq 0$. If $X$ is a solution of (22) then $X=\sigma \gamma$ for some $\gamma \in \mathbf{F}_{22 r}$.

(b) If $\sigma=0$, then any $y, y=L(x)+L(x)^{2^{r}}$ for some $x$, satisfies

$$
\mu^{2^{3 r}} y+\mu^{2^{4 r}} y^{2^{2 r}}=0
$$

and $\mu$ is a $\left(2^{r}+1\right)$ th power.

Proof. We compute $R(x)=L(x)+L(x)^{2^{r}}$ :

$$
\begin{aligned}
R(x) & =\mu x^{2^{r}}+(\mu x)^{2^{4 r}}+\mu^{2^{5 r}}\left(x^{2^{5 r}}+x\right)+\mu^{2^{r}} x^{2^{2 r}}+(\mu x)^{2^{5 r}}+\mu\left(x+x^{2^{r}}\right) \\
& =A x+\mu^{2^{r}} x^{2^{2 r}}+\mu^{2^{4 r}} x^{2^{4 r}},
\end{aligned}
$$

with $A=\left(\mu+\mu^{2^{5 r}}\right)$. Now we compute

$$
\begin{aligned}
M(x) & =\mu^{2^{3 r}} R(x)+\mu^{2^{4 r}} R(x)^{2^{2 r}} \\
& =\left(\mu^{2^{3 r}} A+\mu^{2^{4 r}+1}\right) x+\left(\mu^{2^{3 r}+2^{r}}+A^{2^{2 r}} \mu^{2^{4 r}}\right) x^{2^{2 r}} \\
& =\sigma^{2^{2 r}} x+\sigma x^{2^{2 r}}
\end{aligned}
$$

noticing that $\left(\mu^{2^{3 r}} A+\mu^{2^{4 r}+1}\right)^{2^{4 r}}=\sigma$. We get $M(\sigma)=0$.

If $\sigma \neq 0$ then the kernel of $M$ is exactly $\sigma \mathbf{F}_{2^{2 r}}$. Hence the kernel of $R$ is a subspace of $\sigma \mathbf{F}_{22 r}$. But $\sigma$ is a root of $R$ too: 


$$
\begin{aligned}
& R(\sigma)=\mu^{1+2^{r}+2^{4 r}}+\mu^{1+2^{r}+2^{3 r}}+\mu^{1+2^{2 r}+2^{4 r}} \\
& +\mu^{2^{r}+2^{4 r}+2^{5 r}}+\mu^{2^{r}+2^{3 r}+2^{5 r}}+\mu^{2^{2 r}+2^{4 r}+2^{5 r}} \\
& +\mu^{1+2^{r}+2^{3 r}}+\mu^{2^{r}+2^{3 r}+2^{5 r}}+\mu^{1+2^{r}+2^{4 r}} \\
& +\mu^{2^{2 r}+2^{4 r}+2^{5 r}}+\mu^{2^{r}+2^{4 r}+2^{5 r}}+\mu^{1+2^{2 r}+2^{4 r}} \\
& =0 \text {. }
\end{aligned}
$$

Consequently the kernel of $R$ coincides with $\sigma \mathbf{F}_{2^{2 r}}$. Since the kernel of $L$ is a subspace of the kernel of $R$, then any nonzero solution of (22) belongs to $\sigma \mathbf{F}_{2^{2 r}}^{*}$, completing the proof of (a).

Now suppose that $\sigma=0$, so that $M$ is the null polynomial. Hence any $y=R(x)$ satisfies $\mu^{2^{3 r}} y+\mu^{2^{4 r}} y^{2^{2 r}}=0$, that is, for any such nonzero $y$,

$$
y^{2^{2 r}-1}=\left(\frac{1}{\mu^{2^{r}-1}}\right)^{2^{3 r}} .
$$

This is possible only if $\mu$ is a $\left(2^{r}+1\right)$ th power in $\mathbf{F}_{2^{n}}$, completing the proof.

Now, we are ready to find the Walsh spectrum of $h_{\mu}$, i.e. to determine the dimension of $K(\mu)$ (see Lemma 5).

Theorem 4. Let $\sigma=\mu^{2^{r}+2^{4 r}}+\mu^{2^{r}+2^{3 r}}+\mu^{2^{2 r}+2^{4 r}}$. Then

$$
\operatorname{dim} K(\mu)= \begin{cases}2 r & \text { if } \sigma \neq 0 \\ 4 r & \text { if } \sigma=0\end{cases}
$$

Consequently, the Walsh transform of $h_{\mu}$ takes the values $\left\{0, \pm 2^{5 r}\right\}$ if $\sigma=0$ and $\left\{0, \pm 2^{4 r}\right\}$, otherwise.

Proof. Notation is as in Lemma 8 and in its proof. Consider again the polynomial $R$ which has degree $2^{4 r}$.

When $\sigma \neq 0$, we have shown in the proof of Lemma 8 that the kernel of $R$ has dimension $2 r$. Thus the dimension of the kernel of $L$ is at most $2 r$. By Lemma 7 it holds $\operatorname{dim} K(\mu) \leqslant 3 r$, and Proposition 4 implies $\operatorname{dim} K(\mu)=2 r$.

Assume that $\sigma=0$. By Lemma 8, we know that the image of $R$ is contained in the subspace

$$
J=\{0\} \cup\left\{y \in \mathbf{F}_{2^{n}}, y^{2^{2 r}-1}=\mu^{-2^{3 r}\left(2^{r}-1\right)}\right\},
$$

where $\mu=\beta^{2^{r}+1}$ for some $\beta$. Clearly, we have $J=c \mathbf{F}_{22 r}$ with $c=\beta^{-2^{3 r}}$. Thus the image set of $R$ has dimension at most $2 r$. Therefore, the dimension of the kernel of $R$ is at least $4 r$. Since $R$ has degree $2^{4 r}$, this dimension is exactly $4 r$. Now, the kernel of $L$, say $K_{1}$, is a subspace of the kernel of $R$. Since $R(x)=L(x)+L(x)^{2^{2 r}}$ we get from Lemma 6

$$
\operatorname{dim} K_{1} \in\{3 r, 4 r\}
$$

But $\operatorname{dim} K(\mu)=\operatorname{dim} K_{1}+r$ (see Lemma 7) which leads to $\operatorname{dim} K(\mu)=4 r$, completing the proof. 
Table 2

The exponents $d$, defining bent Boolean functions on $\mathbf{F}_{2^{n}}, n=2 t$, of the form $x \mapsto \operatorname{Tr}\left(\lambda x^{d}\right)$ for some $\lambda \in \mathbf{F}_{2^{n}}$. An exhaustive search shows that there are no other $d$ for $n \leqslant 20$

\begin{tabular}{llll}
\hline Type & Exponent & Condition & References \\
\hline $\mathcal{P} \mathcal{S}_{a p}$ & $a\left(2^{t}-1\right)$ & $\operatorname{gcd}\left(a, 2^{t}+1\right)=1$ & {$[12,16]$} \\
Kasami & $2^{2 i}-2^{i}+1$ & $\operatorname{gcd}(i, n)=1$ & {$[13]$} \\
Maiorana-McFarland & $2^{i}+1$ & $n=\operatorname{gcd}(n, i) s, s$ even & {$[15]$} \\
& $\left(2^{r}+1\right)^{2}$ & $n=4 r$ & {$[9,17]$} \\
& $2^{2 r}+2^{r}+1$ & $n=6 r$ & This paper \\
\hline
\end{tabular}

\section{Conclusions}

The complete classification of monomial bent functions is not achieved. We give in Table 2 the list of known such functions. There are no other for $n \leqslant 20$. Actually, little is known about this corpus, as recalled in [17]. They do not all lie in the known classes, especially in class $\mathcal{M}$. For instance, some bent functions characterized in [13], namely with Kasami exponents, are not normal [4], implying that they do not belong to any previously known class. On the other hand, the most recent results on monomial bent functions provide subclasses of $\mathcal{M}$.

During our work, we investigated general tools for the study of monomial bent functions. Although our proofs, in this paper, seem specific, we introduce several tools for the study of a larger class of Boolean functions, expressed by trace functions, especially those which are of degree 3. Notably, we showed by Example 1 that some functions studied in [17] can be viewed as a concatenation of affine functions. This result will be completed in a forthcoming paper, in a more general context (see $[9,10])$.

The study of functions $f_{\lambda}$ which are not bent leads to several open problems (see Conjecture 1). For this reason, we studied the properties of derivatives of all $f_{\lambda}$. Our study of the functions $g_{a, \lambda}$ can also be placed into the context of the general study of quadratic functions, a topic which is currently discussed [8,23].

\section{References}

[1] T. Berger, A. Canteaut, P. Charpin, Y. Laigle-Chapuy, On almost perfect nonlinear functions, IEEE Trans. Inform. Theory 52 (9) (2006) 4160-4170.

[2] A. Canteaut, P. Charpin, Decomposing bent functions, IEEE Trans. Inform. Theory 49 (8) (2003) $2004-2019$.

[3] A. Canteaut, C. Carlet, P. Charpin, C. Fontaine, On cryptographic properties of the cosets of $R(1, m)$, IEEE Trans. Inform. Theory 47 (4) (2001) 1494-1513.

[4] A. Canteaut, M. Daum, G. Leander, H. Dobbertin, Normal and non normal bent functions, Discr. Appl. Math. 154 (2) (2006) 202-218.

[5] C. Carlet, Recursive lower bounds on the nonlinearity profile of Boolean functions and their applications, Cryptology ePrint Archive, Report 2006/457, http://eprint.iacr.org/, 2006.

[6] C. Carlet, Boolean functions for cryptography and error correcting codes, in: Y. Crama, P. Hammer (Eds.), Boolean Methods and Models, Cambridge Univ. Press, in press.

[7] C. Carlet, P. Gaborit, Hyper-bent functions and cyclic codes, J. Combin. Theory Ser. A 113 (3) (2006) $466-482$.

[8] P. Charpin, C. Tavernier, E. Pasalic, On bent and semi-bent quadratic Boolean functions, IEEE Trans. Inform. Theory 51 (12) (2005) 4287-4298.

[9] P. Charpin, G. Kyureghyan, On cubic bent functions in the class M, in: Proceedings of Algebraic and Combinatorial Coding Theory, ACCT-10, Zvenigorod, Russia, September 2006.

[10] P. Charpin, G. Kyureghyan, Cubic monomial bent functions: A subclass of $\mathcal{M}$, submitted for publication.

[11] P.M. Cohn, Algebra, vol. I, Wiley, 1982.

[12] J. Dillon, Elementary Hadamard difference sets, PhD dissertation, University of Maryland, 1974. 
[13] J. Dillon, H. Dobbertin, New cyclic difference sets with Singer parameters, Finite Fields Appl. 10 (2004) 342-389.

[14] R.W. Fitzgerald, Highly degenerate quadratic forms over finite fields of characteristic 2, Finite Fields Appl. 11 (2005) 165-181.

[15] R. Gold, Maximal recursive sequences with 3-valued recursive crosscorrelation functions, IEEE Trans. Inform. Theory 14 (1) (1968) 154-156.

[16] G. Lachaud, J. Wolfmann, The weights of the orthogonals of the extended quadratic binary Goppa codes, IEEE Trans. Inform. Theory 36 (3) (1990) 686-692.

[17] N.G. Leander, Monomial bent functions, IEEE Trans. Inform. Theory 52 (2) (2006) 738-743.

[18] N.G. Leander, A. Kholosha, Bent functions with $2^{r}$ Niho exponents, IEEE Trans. Inform. Theory 52 (12) (2006) 5529-5532.

[19] R. Lidl, H. Niederreiter, Finite Fields, Cambridge Univ. Press, 1983.

[20] F.J. MacWilliams, N.J.A. Sloane, The Theory of Error Correcting Codes, North-Holland, 1986.

[21] R.J. McEliece, Finite Fields for Computer Scientists and Engineers, Kluwer, 1987.

[22] R.L. McFarland, A family of noncyclic difference sets, J. Combin. Theory Ser. A 15 (1973) 1-10.

[23] N.Y. Yu, G. Gong, Constructions of quadratic bent functions in polynomial forms, IEEE Trans. Inform. Theory 52 (7) (2006) 3291-3299. 